\title{
Integrated analysis of microarray data to identify the genes critical for the rupture of intracranial aneurysm
}

\author{
LIANG WEI*, QI WANG*, YANFEI ZHANG, CHENG YANG, \\ HONGXIN GUAN, JIANXIN JIANG and ZHIYANG SUN
}

Department of Neurosurgery, East Hospital, Tongji University School of Medicine, Shanghai 200120, P.R. China

Received August 1, 2016; Accepted November 24, 2017

DOI: $10.3892 / 01.2018 .7935$

\begin{abstract}
Intracranial aneurysm (IA) is a localized dilation of the blood vessel. The present study was designed to explore the mechanisms of rupture of IA. GSE13353 (including 11 ruptured and 8 unruptured IA samples) and GSE15629 (including 8 ruptured and 6 unruptured IA samples) were downloaded from the Gene Expression Omnibus database. The differentially expressed genes (DEGs) identified using limma and MetaDE packages were merged, and a protein-protein interaction (PPI) network analysis was performed using Cytoscape software. Pathway enrichment analysis was performed for the nodes of the PPI network using the fisher algorithm. The 100 most prominent genes in the network were designated candidate genes and a hierarchical clustering analysis was performed. The tune.svm function of e1071 package was used to construct a support vector machine (SVM) classifier, and the Candidate Cancer Gene Database was applied to analyze the characterization of gene-associated cancer. Furthermore, the genes involved in the SVM classifier were assessed via principal component analysis (PCA). In the ruptured samples, 1,292 DEGs and 1,029 DEGs separately were identified by limma and MetaDE packages. The 100 most prominent genes in the network included fibronectin 1 ( $F N 1$ ), amyloid $\beta$ (A4) precursor protein $(A P P)$, nuclear RNA export factor $1(N X F 1)$ and signal
\end{abstract}

Correspondence to: Dr Zhiyang Sun, Department of Neurosurgery, East Hospital, Tongji University School of Medicine, 150 Jimo Road, Shanghai 200120, P.R. China

E-mail: dfsunzy@126.com

*Contributed equally

Abbreviations: IA, intracranial aneurysm; DEGs, differentially expressed genes; PPI, protein-protein interaction; SVM, support vector machine; PCA, principal component analysis; NF- $\mathrm{BB}$, Nuclear factor- $\kappa \mathrm{B}$; MCP-1, monocyte chemoattractant protein-1; sIA, saccular intracranial aneurysm; BC, betweenness centrality; TLRs, the toll-like receptors

Key words: intracranial aneurysm, differentially expressed genes, protein-protein interaction network, support vector machine, principal component analysis transducer and activator of transcription 3 (STAT3). Pathway enrichment analysis identified that toll-like receptor 3 (TLR3) was enriched in the Toll-like receptor signaling pathway. A total of 15 genes (including $F N 1$ ) were used to construct the SVM classifier. NXF1 was identified to be associated with Nervous System Cancer. PCA revealed that $A P P, N X F 1$ and STAT3 were the 3 principal components. TLR3, FN1, APP, $N X F 1$ and STAT3 may affect the rupture of IA.

\section{Introduction}

As a cerebrovascular disorder, intracranial aneurysm (IA; also designated brain or cerebral aneurysm) is a ballooning or localized dilation of the blood vessel induced by weakness of the artery wall (1). IA cases are divided according to size into small (diameter, $<15 \mathrm{~mm}$ ) and large aneurysms [including large $(15-25 \mathrm{~mm})$, giant $(25-50 \mathrm{~mm})$ and super-giant $(>50 \mathrm{~mm})$ aneurysms] (2). Based on the shape, IA may be classified into saccular aneurysms, fusiform aneurysms and microaneurysms (2). IA may not only be a result of genetic conditions, but also lifestyle factors, including smoking, hypertension, obesity and excess alcohol consumption $(3,4)$. Individuals who are between 30 and 60 years old experience the highest incidence of IA, and women experience an increased incidence compared with men with a ratio of $3: 2(1,5)$.

The genes implicated in the pathogenesis of the rupture of IA have been evaluated previously. Through reverse transcription-quantitative polymerase chain reaction, Guo et al (6) identified that the mRNA levels of caspase-3 in IA and abdominal aortic aneurysm are 8.94-fold and 6.73-fold compared with that of normal vessels, which improves the understanding of apoptosis in ruptured intracranial aneurysm. Nuclear factor- $\kappa \mathrm{B}(\mathrm{NF}-\kappa \mathrm{B})$ functions as an essential regulator during the initiation of IA development via mediation of several inflammatory genes associated with macrophage activation and recruitment, thus, $N F-\kappa B$ may function as a therapeutic target for IA (7). Increased tumor necrosis factor $\alpha$ and Fas-associated death domain protein may have deleterious effects on cerebral arteries through facilitation of inflammation and apoptosis in immune and vascular cells, consequently weakening vessel walls $(8,9)$. Decreased tissue inhibitor of matrix metalloproteinases and increased matrix metalloproteinases in the late stage of IA formation may be the reason for extracellular matrix degradation resulting in the progression, 
and rupture of IA (10). As a main chemoattractant for monocytes and macrophages, NF- $\mathrm{BB}$ activation-induced monocyte chemoattractant protein-1 $(M C P-1)$ acts in IA formation and may serve as a potential target for therapy inhibiting IA progression (11). Nevertheless, the molecular mechanisms of rupture of IA have not been fully revealed.

In 2011, Kurki et al (12) explored the gene expression differences between ruptured and unruptured saccular intracranial aneurysm (sIA) wall samples, screening 686 upregulated and 740 downregulated genes in the ruptured samples, and finding that hypoxia-inducible factor-1A, ETS transcription factors, toll-like receptor signaling and $N F-\kappa B$ are associated with the rupture of sIA walls in humans. In 2010, Pera et al (13) investigated the gene expression profiles of ruptured and unruptured IA, as well as control intracranial arteries, and identified a total of 159 differentially expressed genes (DEGs) and several critical biological processes, including cell adhesion, muscle system, and the immune system, and inflammatory response. However, comprehensive bioinformatic analyses have not been performed to further screen the critical genes associated with the rupture of IA. Using the data gathered by Kurki et al (12) and Pera et al (13), the DEGs between ruptured, and unruptured IA samples were fully screened. Utilizing protein-protein interaction (PPI) network construction, pathway enrichment analysis, construction and efficiency evaluation of support vector machine (SVM) classifier, and principal component analysis (PCA), the key genes implicated in the rupture of IA were further identified.

\section{Materials and methods}

Microarray data. Microarray data of GSE13353 (https://www. ncbi.nlm.nih.gov/geo/query/acc.cgi?acc=GSE13353) and GSE15629 (https://www.ncbi.nlm.nih.gov/geo/query/acc. cgi?acc=GSE15629) were downloaded from the Gene Expression Omnibus database. GSE13353, which was deposited by Kurki et al (12) and sequenced on the platform of GPL570 (HG-U133_Plus_2) Affymetrix Human Genome U133 Plus 2.0 Array, included 11 ruptured IA wall samples and 8 unruptured IA wall samples. Kurki et al (12) isolated ruptured and unruptured IA wall samples from the necks of Finnish individuals undergoing microsurgical clipping as previously described (14-17). The IA wall samples were frozen in liquid nitrogen and then kept in the Helsinki Neurosurgery sIA Tissue Bank (12). GSE15629 was deposited by Pera et al (13) and sequenced on the platform of GPL6244 (HuGene-1_0-st) Affymetrix Human Gene 1.0 ST Array [transcript (gene) version], from which 8 ruptured IA wall samples and 6 unruptured IA wall samples were selected for this study. Pera et al (13) also resected full-thickness vessel wall samples from patients who underwent microsurgical clipping. Then, the samples were stored in RNAlater (Qiagen China Co., Ltd., Shanghai, China) at $80^{\circ} \mathrm{C}$.

Data preprocessing and DEGs screening. Using the oligo package (18) in $\mathrm{R}$, background correction and normalization were performed on the raw data. Using the limma package (http://www.R-project.org) (19) in R, the genes differentially expressed between ruptured and unruptured IA samples were selected, with $\mathrm{P}<0.05$ and Ifold change $\mathrm{I}>1.5$ as the thresholds.
Conversely, based on the MetaDE package (https://cran.r-project. org/web/packages/MetaDE/index.html) (20) in R, heterogeneity tests and differential expression analysis for each gene were conducted successively. The genes with $\tau^{2}=0$ and Qpval $>0.05$ were homogeneous and unbiased, from which the genes with $\mathrm{P}<0.05$ were further selected as DEGs.

Construction of PPI network. The interaction information of human proteins was downloaded from the Biological General Repository for Interaction Datasets (http://thebiogrid.org/) (21), Human Protein Reference Database (http://www.hprd.org/) (22) and Database of Interacting Proteins (http://dip.doe-mbi.ucla.edu/) (23) databases. Subsequently, the identified DEGs were mapped to the interaction network of human proteins and the PPI network for the DEGs was visualized using Cytoscape software (version 3.1.0, http://www.cytoscape.org) (24). In the PPI network, nodes and edges separately stand for proteins, and interactions of proteins. Additionally, the number of edges involving one node was the connectivity degree of the node. Furthermore, the candidate genes in the PPI network were further screened using the betweenness centrality (BC) method based on the following formula:

$$
\mathrm{C}_{\mathrm{B}}(v)=\sum_{t \neq v \neq u \in V} \frac{\sigma_{s t}(v)}{\sigma_{s t}}
$$

$\sigma_{s t}$ represents the number of the shortest path between $\mathrm{s}$ and t. $\sigma_{s t}(v)$ stands for the number of paths past node $\mathrm{v}$ among the shortest paths between $s$ and $t$. The value of $C_{B}$ (v) ranges from 0 to 1 , a larger value indicates an increased importance.

Additionally, hierarchical clustering analysis (25) was performed for the candidate genes and clustering results were visualized using Heatmap software (version 1.0, http://www.hiv. lanl.gov/content/sequence/HEATMAP/heatmap.html) (26).

Pathway enrichment analysis. Pathway enrichment analysis was performed for the DEGs involved in the PPI network using the fisher algorithm (27) based on the following formula:

$$
p=1-\sum_{i=0}^{x-1} \frac{\left(\begin{array}{l}
M \\
i
\end{array}\right)\left(\begin{array}{l}
N-M \\
K-i
\end{array}\right)}{\left(\begin{array}{l}
N \\
K
\end{array}\right)}
$$

Among the formula, $\mathrm{M}, \mathrm{N}$ and $\mathrm{K}$ stand for the number of genes enriched in pathways, the total number of genes in whole genome and the number of DEGs, respectively. $p$ represents the probability that no less than $x$ DEGs are enriched in pathways.

Construction and efficiency evaluation of SVM classifier. As effective classifiers, SVMs may be utilized for two-class classification of microarray data and acquire high classification accuracy (28). The DEGs involved in the PPI network were sorted in descending order based on their BC values. Starting from the last 10 genes and taking 5 genes as an interval, the DEGs were selected as characterization factors 


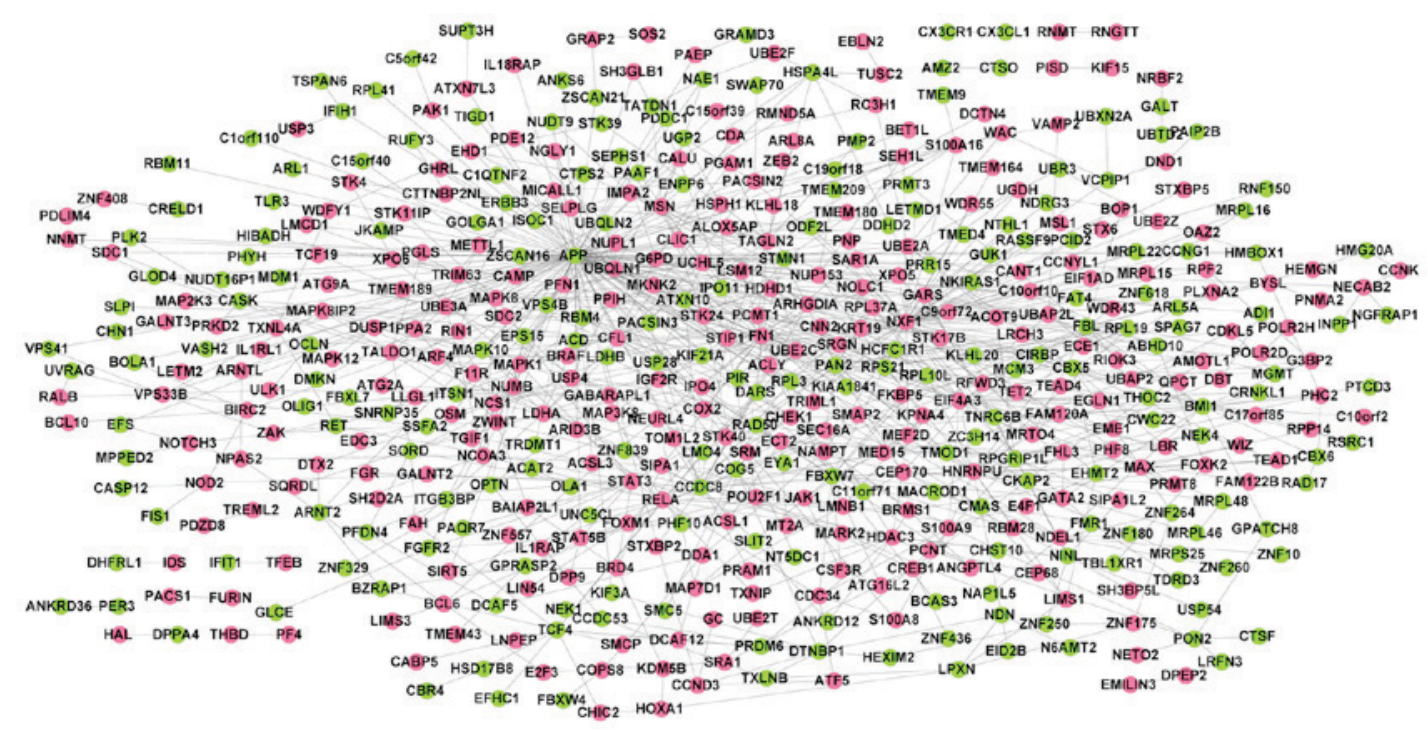

Figure 1. Protein-protein interaction network for the merged differentially expressed genes. Red and green nodes represent upregulated and downregulated genes in the ruptured samples, respectively.

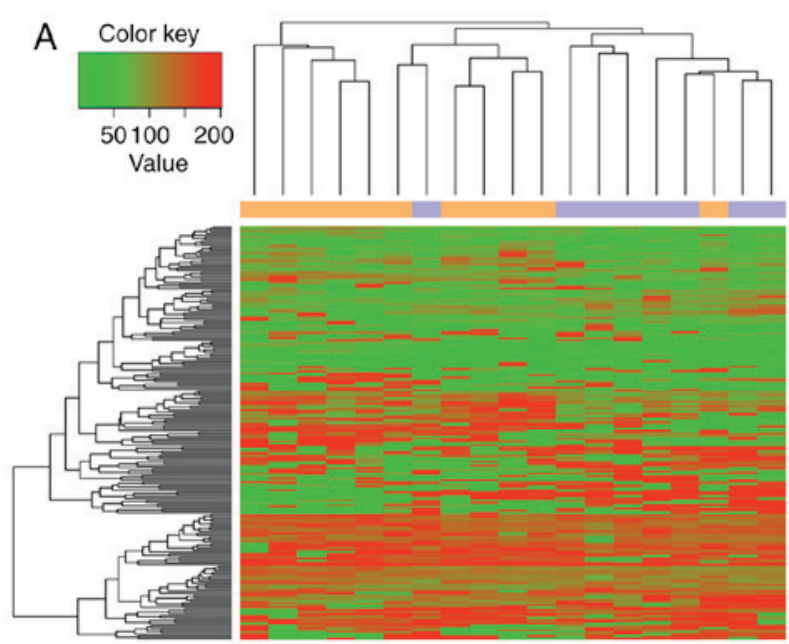

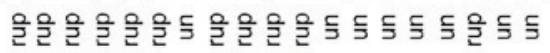

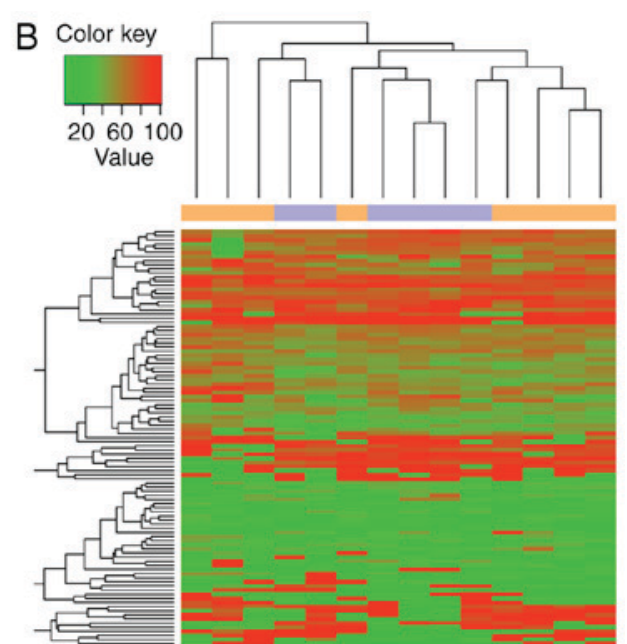

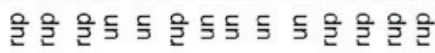

Figure 2. Hierarchical clustering trees for (A) GSE13353 and (B) GSE15429. Red and green stand for high and low values, respectively. Rup, ruptured; un, unruptured.

(genes used for constructing SVM classifier) in descending order based on BC values. With the dataset of GSE13353 as the training dataset, the tune.svm function of the e1071 package (http://cran.r-project.org/web/packages/e1071/) (29) in $\mathrm{R}$ was used to construct an optimal SVM classifier. Then, the dataset of GSE15629 was used as the validation dataset for detecting the SVM classifier. Furthermore, the Candidate Cancer Gene Database (http://ccgd-starrlab.oit. umn.edu/) (30) was used to search for the characterization of gene-associated cancer.

PCA of characterization factors. As a multivariate technique, PCA is able to reassemble associated-indexes into a new set of comprehensive indexes that exhibit no correlations with each other (31). To further optimize the identified characterization genes, the genes involved in the SVM classifier were performed using PCA.

\section{Results}

DEGs analysis. In the ruptured samples, a total of 636 DEGs (including 279 upregulated genes and 357 downregulated genes) in GSE13353 and 656 DEGs (including 130 upregulated genes and 526 downregulated genes) in GSE15629 were identified using the limma package. Using the MetaDE package, a total of 1,029 DEGs (including 527 upregulated genes and 502 downregulated genes) were screened. To include the associated genes into our research, the DEGs identified by limma and MetaDE packages were merged and used for the following analyses.

PPI network analysis and pathway enrichment analysis. The PPI network constructed for the merged DEGs identified 510 nodes (including 290 upregulated genes and 220 downregulated genes) and 907 interactions (Fig. 1). Combined 
Table I. Pathways enriched for the genes involved in the protein-protein interaction network.

\begin{tabular}{|c|c|c|c|}
\hline Description & Gene number & P-value & Gene symbol \\
\hline $\begin{array}{l}\text { hsa04010: MAPK } \\
\text { signaling pathway }\end{array}$ & 18 & 0.004304 & $\begin{array}{l}F G F R 2, Z A K, B R A F, M A P 2 K 3, \text { RELA, MKNK2, } \\
\text { MAPK10, STK4, MAX, MAPK1, MAPK12, DUSP1, } \\
\text { MAP3K8, SOS2, MAPK8IP2, MAPK8, PAK1, STMN1 }\end{array}$ \\
\hline $\begin{array}{l}\text { hsa05200: Pathways } \\
\text { in cancer }\end{array}$ & 20 & 0.007181 & $\begin{array}{l}F G F R 2, E 2 F 3, R E T, B R A F, R E L A, S T A T 5 B, A R N T 2, \\
E G L N 1, \text { MAPK10, STK4, BIRC2, STAT3, MAPK1, } \\
M A X, S O S 2, R A L B, J A K 1, C S F 3 R, M A P K 8, F N 1\end{array}$ \\
\hline $\begin{array}{l}\text { hsa04621: NOD-like } \\
\text { receptor signaling pathway }\end{array}$ & 7 & 0.013053 & $\begin{array}{l}\text { MAPK1, NOD2, MAPK12, RELA, MAPK8, MAPK10, } \\
\text { BIRC2 }\end{array}$ \\
\hline $\begin{array}{l}\text { hsa04012: ErbB signaling } \\
\text { pathway }\end{array}$ & 8 & 0.019615 & $\begin{array}{l}\text { MAPK1, BRAF, ERBB3, STAT5B, SOS2, MAPK8, } \\
\text { MAPK10, PAK1 }\end{array}$ \\
\hline $\begin{array}{l}\text { hsa00270: Cysteine and } \\
\text { methionine metabolism }\end{array}$ & 5 & 0.021434 & $A D I 1, L D H B, L D H A, S R M, T R D M T 1$ \\
\hline $\begin{array}{l}\text { hsa04620: Toll-like receptor } \\
\text { signaling pathway }\end{array}$ & 8 & 0.040118 & $\begin{array}{l}\text { MAPK1, MAPK12, RELA, MAP } 2 K 3, \text { MAP } 3 K 8, T L R 3, \\
\text { МАРK8, MAPK10 }\end{array}$ \\
\hline $\begin{array}{l}\text { hsa04722: Neurotrophin } \\
\text { signaling pathway }\end{array}$ & 9 & 0.042021 & $\begin{array}{l}\text { MAPK1, MAPK12, BRAF, RELA, SOS2, NGFRAP1, } \\
\text { MAPK8, MAPK10, ARHGDIA }\end{array}$ \\
\hline
\end{tabular}
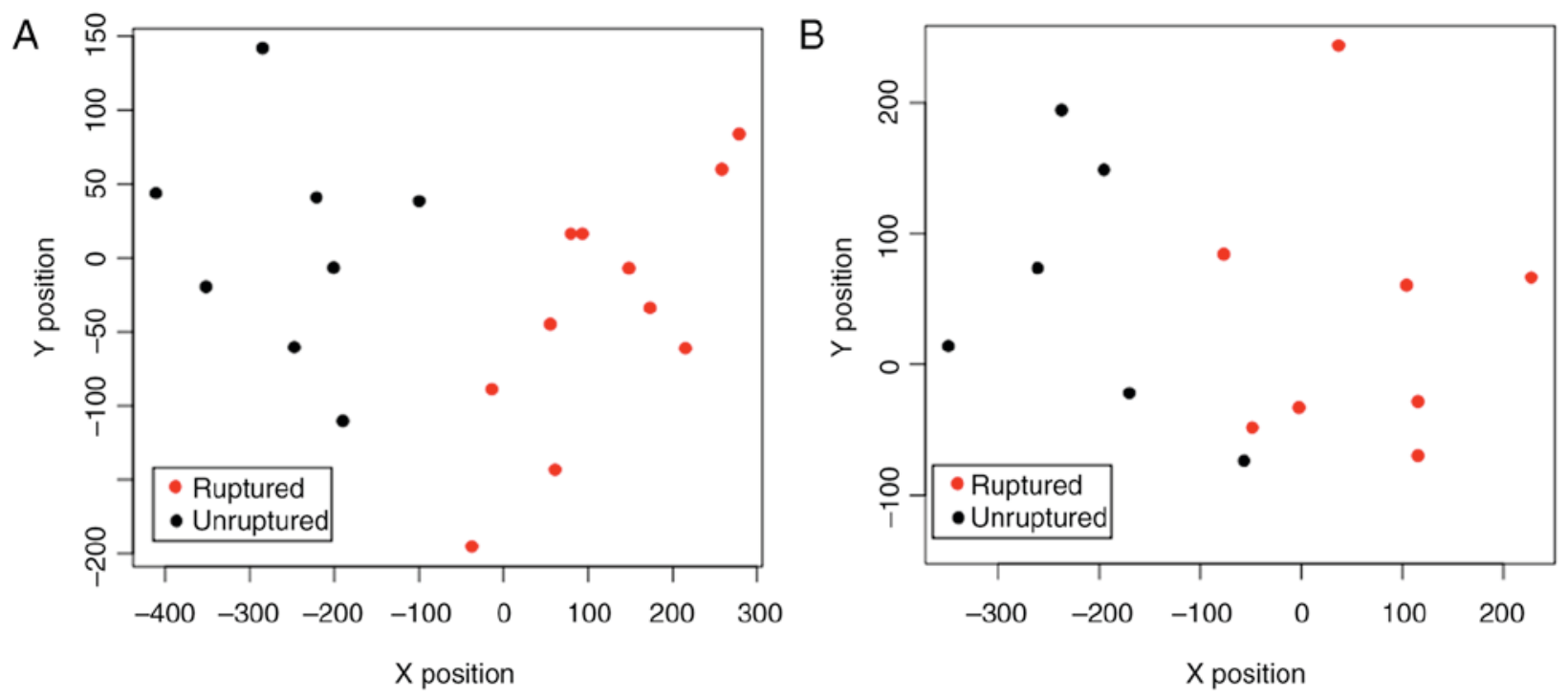

Figure 3. Support vector machine classifier was able to fully differentiate the samples in (A) the training dataset of GSE13353 and (B) the validation dataset of GSE15629. Red and black dots represent ruptured and unruptured samples respectively. X and Y axis indicate the position vectors of samples.

with connectivity degrees and BC scores, the top 100 genes [including fibronectin 1 (FN1), amyloid $\beta$ (A4) precursor protein (APP), nuclear RNA export factor 1 (NXF1) and signal transducer and activator of transcription 3 (STAT3)] in the PPI network were selected as candidate genes. Hierarchical clustering analysis revealed that the candidate genes were able to separate the ruptured samples from the unruptured samples (Fig. 2).

To further understand the biological pathways involved the genes in PPI network, pathway enrichment analysis was conducted. A total of 7 pathways were enriched, including the mitogen-activated protein kinase signaling pathway $(\mathrm{P}=0.004304)$, pathways in cancer $(\mathrm{P}=0.007181)$ and Toll-like receptor signaling pathway $(\mathrm{P}=0.040118)$, which involved toll-like receptor 3 (TLR3) (Table I).
Construction and efficiency evaluation of SVM classifier. With the dataset of GSE13353 as the training dataset, optimization of the SVM classifier was performed. When the number of characterization genes reached 15, the SVM classifier could completely and accurately distinguish all samples (Fig. 3A). Thus, the 15 genes (including FN1) were considered as the genes required for constructing the SVM classifier (parameters: $\gamma, 0.5$; cost, 4 ; cross, 10). To confirm that the SVM classifier had repeatability and portability, the validation dataset of GSE15629 was used to detect the SVM classifier. In the dataset of GSE15629, the SVM classifier was able to fully differentiate the ruptured samples from the unruptured samples (Fig. 3B). Therefore, the expression pattern characteristics of the 15 characterization genes in ruptured and unruptured samples were notable. Furthermore, 
Table II. The 15 characterization genes involved in the support vector machine classifier and the types of cancer associated with them.

\begin{tabular}{|c|c|c|c|c|c|c|}
\hline Gene & BC_score & Degree & P-value & $\log \mathrm{FC}$ & Pubmed ID & Cancer type \\
\hline$A P P$ & 0.9309 & 113 & 0.022006 & -1.40543 & 22057237 & Colorectal cancer \\
\hline BMII & 0.5413 & 19 & 0.000794 & -1.21654 & & \\
\hline$C C D C 8$ & 0.5700 & 26 & 0.003424 & -1.04234 & & \\
\hline CFLL1 & 0.5344 & 14 & 0.049459 & 0.817437 & & \\
\hline$F B L$ & 0.5457 & 17 & 0.032831 & -0.77802 & & \\
\hline$F B X W 7$ & 0.5380 & 19 & 0.024609 & -1.45185 & 22370638 & Blood cancer \\
\hline FN1 & 0.6292 & 42 & 0.01076 & 1.299781 & & \\
\hline$H N R N P U$ & 0.5447 & 24 & 0.049798 & 0.906204 & 27006499 & Gastric cancer \\
\hline NDEL1 & 0.5339 & 10 & 0.036456 & 0.840461 & 24316982 & Liver cancer \\
\hline$N X F 1$ & 0.7250 & 57 & 0.004859 & 1.123627 & 23685747 & Nervous system cancer \\
\hline$R E L A$ & 0.5382 & 18 & 0.037826 & 0.860905 & & \\
\hline STAT3 & 0.5487 & 15 & 0.00697 & 1.266099 & 22699621 & Pancreatic cancer \\
\hline$T A G L N 2$ & 0.5337 & 12 & 0.000581 & 1.101816 & & \\
\hline TCF4 & 0.5357 & 12 & 0.045941 & -0.95848 & 23045694 & Nervous system cancer \\
\hline$U B Q L N 1$ & 0.5464 & 16 & 0.016495 & 0.942896 & 24316982 & Liver cancer \\
\hline
\end{tabular}

BC, betweenness centrality; FC, fold change; ID, identification.

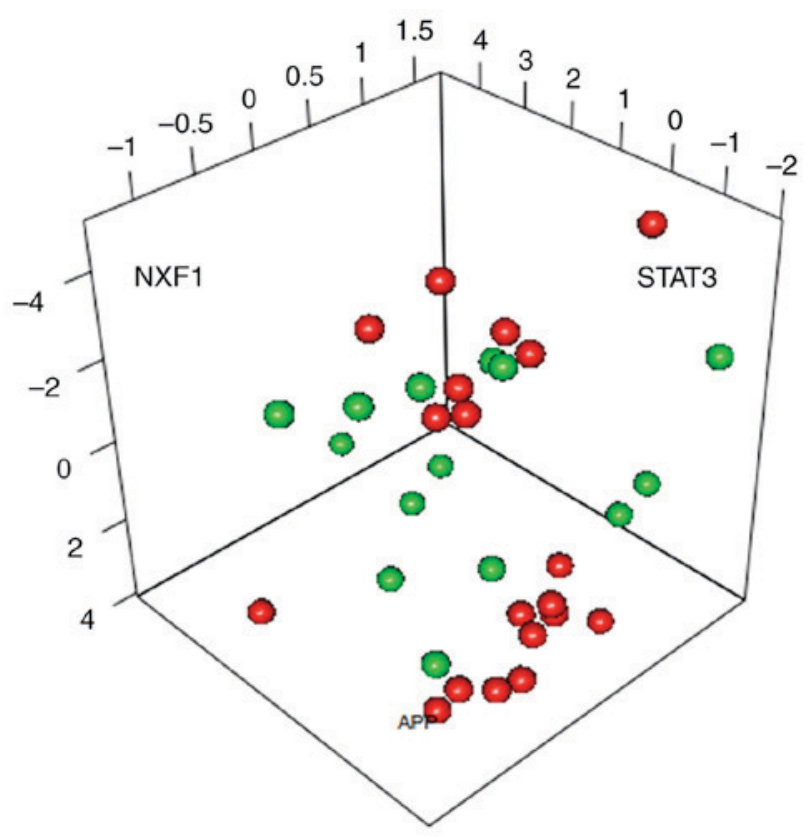

Figure 4. Three-dimensional diagram identifying that the three principal components may be able to separate the samples. Red and green circles represent ruptured and unruptured intracranial aneurysm samples, respectively NXF1, nuclear RNA export factor 1; STAT3, signal transducer and activator of transcription 3; APP, amyloid $\beta$ (A4) precursor protein.

the characterization of gene-associated cancer was assessed, revealing that $N X F 1$ was associated with nervous system cancer (Table II).

PCA of characterization factors. To further optimize the identified characterization genes, PCA was conducted on the 15 characterization genes involved in the SVM classifier.
APP, NXF1 and STAT3 were the 3 principal components able to separate the samples (Fig. 4).

\section{Discussion}

In the present study, the DEGs between ruptured and unruptured samples were identified using limma and MetaDE packages. In the ruptured samples, a total of 636 DEGs in GSE13353 and 656 DEGs in GSE15629 were identified by the limma package. Using the MetaDE package, a total of 1,029 DEGs were screened. These DEGs were merged and a PPI network analysis was performed. Combined with connectivity degrees and $\mathrm{BC}$ scores, the top 100 genes (including FN1, APP, NXF1 and STAT3) in the PPI network were identified as candidate genes.

Pathway enrichment analysis of the genes in the PPI network revealed that TLR3 was enriched in the TLR signaling pathway. TLRs have been reported to function in vascular inflammatory diseases including aneurysm and atherosclerosis (32). TLR4, which is expressed in IA walls of humans and rats, may be implicated in IA formation via NF- $\kappa \mathrm{B}$ activation in endothelial cells (33). FN1 was one of the 15 genes used for constructing the SVM classifier. Alternatively spliced extra domain A (EDA) of fibronectin is essential for tissue repair, and decreased expression of EDA may promote susceptibility to aneurysm of patients with a bicuspid aortic valve (34). Wang and Astrof (35) demonstrated that the local synthesis of FN1 serves essential functions in spatial regulation of Notch signaling and cardiovascular development. As a multi-domain extracellular matrix glycoprotein, fibronectin functions in blood vessel morphogenesis during pathological angiogenesis and embryonic development, and is expressed during pathological angiogenesis in multiple diseases, including late stage artherosclerosis, lung cancer and in abnormal ocular conditions (36-39). Thus, TLR3 and FN1 may serve functions in the rupture of IA. 
PCA identified that APP, NXF1 and STAT3 were the three principal components that were able to separate the samples. Duplication of the APP locus, which leads to the increase of amyloid- $\beta$ peptides, is able to induce autosomal dominant early-onset Alzheimer's disease with cerebral amyloid angiopathy (40). As a result of assessing the characterization of gene-associated cancer, $N X F 1$ was identified to be associated with nervous system cancer. $N X F 1$, which belongs to a family of evolutionarily conserved proteins, includes an NTF2-like domain, a noncanonical RNP-type RNA binding domain, a ubiquitin-associated domain and four leucine-rich repeats (41). In vivo blockade of STAT3 signaling or inhibition of interleukin-17A $(I L-17 \mathrm{~A})$ lead to an apparent increase in fatal rupture and aneurysm severity in mouse models, and the prevalence of vascular abnormalities are high in patients with STAT3 deficiency (42). Romain et al (43) identified that T cell-specific STAT3 signaling serves a central function in promoting vascular aneurysm and $I L-17$ serves a protective function in the process. Phosphorylated STAT3 is associated with TLR4-dependent abdominal aortic aneurysm (AAA) formation, and STAT3 and/or TLR4 may be utilized for therapy of AAA $(44,45)$. The level of phosphorylated STAT1 increases during aneurysmal degeneration, and the loss of STATl is associated with aneurysm formation and an increased rate of aortic rupture in a model of aortic dissection (46). These findings indicate that APP, NXF1 and STAT3 may be involved in the rupture of IA.

In conclusion, the DEGs between ruptured and unruptured samples were identified using limma, and MetaDE packages. Additionally, TLR3, FN1, APP, NXF1 and STAT3 may function in the rupture of IA. However, a further validation of the roles of these genes in the rupture of IA is required.

\section{Acknowledgements}

The present study was supported by Shanghai Municipal Commission of Health and Family Planning (grant no. 201440319). The authors would like to thank to Fenghe (Shanghai) Information Technology Co., Ltd for their help.

\section{References}

1. Brisman JL, Song JK and Newell DW: Cerebral aneurysms. N Eng J Med 355: 928-939, 2006.

2. Bhidayasiri R, Waters MF and Giza C: Neurological differential diagnosis: A prioritized approach. John Wiley \& Sons, pp560, 2005.

3. Goljan EF (ed): Rapid Review Pathology. 3rd edition. Mosby Inc., Maryland Heights, MO, 2011.

4. Flemming KD: Stroke essentials for primary care: A practical guide. Mayo Clin Proc 85: e76, 2010.

5. Haberland C: Clinical Neuropathology. Text and Color Atlas. 1 edition. Demos Medical, 2006.

6. Guo F, Li Z, Song L, Han T, Feng Q, Guo Y, Xu J, He M and You C: Increased apoptosis and cysteinyl aspartate specific protease-3 gene expression in human intracranial aneurysm. J Clin Neurosci 14: 550-555, 2007.

7. Aoki T, Kataoka H, Shimamura M, Nakagami H, Wakayama K, Moriwaki T, Ishibashi R, Nozaki K, Morishita R and Hashimoto N: NF-kappaB is a key mediator of cerebral aneurysm formation. Circulation 116: 2830-2840, 2007.

8. Jayaraman T, Berenstein V, Li X, Mayer J, Silane M, Shin YS, Niimi Y, Kiliç T, Gunel M and Berenstein A: Tumor necrosis factor $\alpha$ is a key modulator of inflammation in cerebral aneurysms. Neurosurgery 57: 558-564, 2005.
9. Fontanella M, Rainero I, Gallone S, Rubino E, Fenoglio P, Valfrè W, Garbossa D, Carlino C, Ducati A and Pinessi L: Tumor necrosis factor-alpha gene and cerebral aneurysms. Neurosurgery 60: 668-673, 2007.

10. Aoki T, Kataoka H, Moriwaki T, Nozaki K and Hashimoto N: Role of TIMP-1 and TIMP-2 in the progression of cerebral aneurysms. Stroke 38: 2337-2345, 2007.

11. Aoki T, Kataoka H, Ishibashi R, Nozaki K, Egashira K and Hashimoto N: Impact of monocyte chemoattractant protein-1 deficiency on cerebral aneurysm formation. Stroke 40: 942-951, 2009.

12. Kurki MI, Häkkinen SK, Frösen J, Tulamo R, von und zu Fraunberg M, Wong G, Tromp G, Niemelä M, Hernesniemi J, Jääskeläinen JE and Ylä-Herttuala S: Upregulated signaling pathways in ruptured human saccular intracranial aneurysm wall: An emerging regulative role of toll-like receptor signaling and nuclear factor- $\kappa \mathrm{B}$, hypoxia-inducible factor-1A, and ETS transcription factors. Neurosurgery 68: 1667-1676, 2011

13. Pera J, Korostynski M, Krzyszkowski T, Czopek J, Slowik A, Dziedzic T, Piechota M, Stachura K, Moskala M, Przewlocki R and Szczudlik A: Gene expression profiles in human ruptured and unruptured intracranial aneurysms: What is the role of inflammation? Stroke 41: 224-231, 2010.

14. Frösen J, Piippo A, Paetau A, Kangasniemi M, Niemelä M, Hernesniemi $\mathbf{J}$ and Jääskeläinen $\mathrm{J}$ : Remodeling of saccular cerebral artery aneurysm wall is associated with rupture: Histological analysis of 24 unruptured and 42 ruptured cases. Stroke 35: 2287-2293, 2004.

15. Frösen J, Piippo A, Paetau A, Kangasniemi M, Niemelä M, Hernesniemi J and Jääskeläinen J: Growth factor receptor expression and remodeling of saccular cerebral artery aneurysm walls: Implications for biological therapy preventing rupture. Neurosurgery 58: 534-541, 2006.

16. Tulamo R, Frösen J, Junnikkala S, Paetau A, Pitkäniemi J, Kangasniemi M, Niemelä M, Jääskeläinen J, Jokitalo E, Karatas A, et al: Complement activation associates with saccular cerebral artery aneurysm wall degeneration and rupture. Neurosurgery 59: 1069-1077, 2006.

17. Laaksamo E, Tulamo R, Baumann M, Dashti R, Hernesniemi J, Juvela $S$, Niemelä $M$ and Laakso A: Involvement of mitogen-activated protein kinase signaling in growth and rupture of human intracranial aneurysms. Stroke 39: 886-892, 2008.

18. Rao Y, Lee Y, Jarjoura D, Ruppert AS, Liu CG, Hsu JC and Hagan JP: A comparison of normalization techniques for microRNA microarray data. Stat Appl Genet Mol Biol 7: Article 22, 2008.

19. Smyth GK: Limma: Linear models for microarray data. In: Bioinformatics and computational biology solutions using R and Bioconductor. Springer, New York, NY, pp397-420, 2005.

20. Chang LC, Lin HM, Sibille E and Tseng GC: Meta-analysis methods for combining multiple expression profiles: Comparisons, statistical characterization and an application guideline. BMC Bioinformatics 14: 368, 2013.

21. Stark C, Breitkreutz BJ, Reguly T, Boucher L, Breitkreutz A and Tyers M: BioGRID: A general repository for interaction datasets. Nucleic Acids Res 34 (Database Issue): D535-D539, 2006.

22. Keshava Prasad TS, Goel R, Kandasamy K, Keerthikumar S, Kumar S, Mathivanan S, Telikicherla D, Raju R, Shafreen B, Venugopal A, et al: Human protein reference database-2009 update. Nucleic Acids Res 37 (Database Issue): D767-D772, 2009.

23. Xenarios I, Rice DW, Salwinski L, Baron MK, Marcotte EM and Eisenberg D: DIP: The database of interacting proteins. Nucleic Acids Res 28: 289-291, 2000.

24. Kohl M, Wiese S and Warscheid B: Cytoscape: Software for visualization and analysis of biological networks. Methods Mol Biol 696: 291-303, 2011.

25. Köhn HF and Hubert LJ: Hierarchical cluster analysis. Wiley StatsRef: Statistics Reference Online, 2015.

26. Wilkinson L and Friendly M: The history of the cluster heat map. Am Stat 63: 179-184, 2012.

27. Damar İH, Altunkaş F, Çelik A, Koç F, Karayakalı M, Karaman K, Arssoy A and Ceyhan K: Fragmented QRS frequency in patients with cardiac syndrome X. Anatol J Cardiol 16: 616-620, 2016.

28. Ma S, Lv M, Deng F, Zhang X, Zhai H and Lv W: Predicting the ecotoxicity of ionic liquids towards Vibrio fischeri using genetic function approximation and least squares support vector machine. J Hazard Mater 283: 591-598, 2015.

29. Dimitriadou E, Hornik K, Leisch F, Meyer D and Weingessel A: Misc functions of the Department of Statistics (e1071), TU Wien. R package 1: 5-24, 2008. 
30. Abbott KL, Nyre ET, Abrahante J, Ho YY, Vogel RI and Starr TK The candidate cancer gene database: A database of cancer driver genes from forward genetic screens in mice. Nucleic Acids Res 43 (Database Issue): D844-D848, 2015.

31. Abdi $\mathrm{H}$ and Williams LJ: Principal component analysis. Wiley Interdisciplinary Reviews: Comput Stat 2: 433-459, 2010.

32. Huggins C, Pearce S, Peri F, Neumann F, Cockerill G and Pirianov G: A novel small molecule TLR4 antagonist (IAXO-102) negatively regulates non-hematopoietic toll like receptor 4 signalling and inhibits aortic aneurysms development. Atherosclerosis 242: 563-570, 2015.

33. Aoki T, Nishimura M, Ishibashi R, Kataoka H, Takagi $Y$ and Hashimoto N: Toll-like receptor 4 expression during cerebral aneurysm formation. Laboratory investigation. J Neurosurg 113: 851-858, 2010

34. Paloschi V, Kurtovic S, Folkersen L, Gomez D, Wågsäter D, Roy J, Petrini J, Eriksson MJ, Caidahl K, Hamsten A, et al: Impaired splicing of fibronectin is associated with thoracic aortic aneurysm formation in patients with bicuspid aortic valve. Arterioscler Thromb Vasc Biol 31: 691-697, 2011.

35. Wang $X$ and Astrof $S$ : Neural crest cell-autonomous roles of fibronectin in cardiovascular development. Development 143: 88-100, 2016.

36. Astrof S and Hynes RO: Fibronectins in vascular morphogenesis. Angiogenesis 12: 165-175, 2009.

37. Neri D and Bicknell R: Tumour vascular targeting. Nat Rev Cancer 5: 436-446, 2005.

38. Pedretti M, Rancic ZA, Herzog BA, Soltermann A, Herzog BA, Schliemann C, Lachat M, Neri D and Kaufmann PA: Comparative immunohistochemical staining of atherosclerotic plaques using F16, F8 and L19: Three clinical-grade fully human antibodies. Atherosclerosis 208: 382-389, 2010.

39. Pedretti M, Soltermann A, Arni S, Weder W, Neri D and Hillinger S: Comparative immunohistochemistry of L19 and F16 in non-small cell lung cancer and mesothelioma: Two human antibodies investigated in clinical trials in patients with cancer. Lung Cancer 64: 28-33, 2009.

40. Rovelet-Lecrux A, Hannequin D, Raux G, Le Meur N, Laquerrière A, Vital A, Dumanchin C, Feuillette S, Brice A, Vercelletto M, et al: APP locus duplication causes autosoma dominant early-onset Alzheimer disease with cerebral amyloid angiopathy. Nat Genet 38: 24-26, 2006.
41. Herold A, Suyama M, Rodrigues JP, Braun IC, Kutay U, Carmo-Fonseca M, Bork P and Izaurralde E: TAP (NXF1) belongs to a multigene family of putative RNA export factors with a conserved modular architecture. Mol Cell Biol 20: 8996-9008, 2000.

42. Chandesris MO, Azarine A, Ong KT, Taleb S, Boutouyrie P, Mousseaux E, Romain M, Bozec E, Laurent S, Boddaert N, et al: Frequent and widespread vascular abnormalities in human signal transducer and activator of transcription 3 deficiency. Circ Cardiovasc Genet 5: 25-34, 2012.

43. Romain M, Taleb S, Dalloz M, Ponnuswamy P, Esposito B, Pérez N, Wang Y, Yoshimura A, Tedgui A and Mallat Z: Overexpression of SOCS3 in T lymphocytes leads to impaired interleukin-17 production and severe aortic aneurysm formation in mice-brief report. Arterioscler Thromb Vasc Biol 33: 581-584, 2013.

44. Liao M, Xu J, Clair AJ, Ehrman B, Graham LM and Eagleton MJ: Local and systemic alterations in signal transducers and activators of transcription (STAT) associated with human abdominal aortic aneurysms. J Surg Res 176: 321-328, 2012.

45. Qin Z, Bagley J, Sukhova G, Baur WE, Park HJ, Beasley D, Libby P, Zhang Y and Galper JB: Angiotensin II-induced TLR4 mediated abdominal aortic aneurysm in apolipoprotein E knockout mice is dependent on STAT3. J Mol Cell Cardiol 87: 160-170, 2015.

46. Eagleton MJ, Xu J, Liao M, Parine B, Chisolm GM and Graham LM: Loss of STAT1 is associated with increased aortic rupture in an experimental model of aortic dissection and aneurysm formation. J Vasc Surg 51: 951-961, 2010.

This work is licensed under a Creative Commons Attribution-NonCommercial-NoDerivatives 4.0 International (CC BY-NC-ND 4.0) License. 